\title{
Estimating Road Vehicle Instantaneous Fuel Consumption by Aerial Traffic Observation with a Multi-Rotor Drone
}

\author{
C.M. Soon ${ }^{1}$, M.A. Abas ${ }^{1}$, S.F. Zainal Abidin² and W.S.I. Wan Salim ${ }^{3}$ \\ ${ }^{1}$ Automotive Development Centre, Institute for Vehicle System and Engineering, Universiti Teknologi Malaysia, 81300 Johor Bahru, Johor, Malaysia \\ ${ }^{2}$ Centre of Automotive and Powertrain Technology, Universiti Tun Hussein Onn Malaysia, 84600 Panchor, Johor, Malaysia \\ ${ }^{3}$ Faculty of Mechanical and Manufacturing Engineering, Universiti Tun Hussein Onn Malaysia, 86400 Parit Raja, Johor, Malaysia
}

\begin{abstract}
This study presents a preliminary approach to estimate instantaneous fuel consumption base on image processing from aerial observation using a multi-rotor drone. A drone was deployed over an actual road traffic to capture images of vehicle activities and feed into a program that was developed in this study. The program identifies and tracks the vehicle activities using pixel-based adaptive approach. The vehicle activities were then processed into variables as an input for the generic vehicle model. Coupled with model constants, the generic vehicle model then estimates the instantaneous fuel consumption and $\mathrm{CO}_{2}$ emission and tags the estimated results on the tracked vehicle on the program user-interface. In comparison with the actual experimental measurements, the estimated instantaneous fuel consumption shows a trend with correlation coefficient of 0.741 with higher total fuel usage by $10.6 \%$. The estimation results were useful to map the distribution of fuel consumption over the routes of the observed area in relation to the natural traffic.
\end{abstract}

\section{ARTICLE HISTORY}

Received: 16 ${ }^{\text {th }}$ Sept 2021

Revised: $4^{\text {th }}$ Oct 2021

Accepted: 10 $0^{\text {th }}$ Nov 2021

\section{KEYWORDS}

Fuel consumption

Emission

Image processing

Vehicle modelling

Drone

\section{INTRODUCTION}

Driven largely by increased of $\mathrm{CO}_{2}$ and other emissions as a result from human activities into the atmosphere, the average surface temperature on earth has constantly rising since the late $19^{\text {th }}$ century [1] and the atmospheric concentrations of $\mathrm{CO}_{2}$ in 2017 has surpassed the $410 \mathrm{ppm}$, highest level in the last three million years [2]. From the $\mathrm{CO} 2$ growth, transportation contributed $25 \%$ which is the second largest contributor and is expected to further increase [3]. Therefore, it is crucial to respond through mitigation and adaptation actions at the earliest else it would lead to irreversible changes to the environment.

Measuring and estimating road transport activities have always been among the studies being conducted to further understand the characteristics of fuel consumption and productivity of pollutant emissions. Concurrently, efforts have been conducted relentlessly to further improve the measurements and estimation methods through many aspects, from the data collection to the data analysis. Among the known measurement approaches are remote sensing [4], Portable Emissions Measurement Systems (PEMS) [5] and chassis dynamometer testing [6]. Besides the measurement, fuel consumption and pollutant estimation are also being estimated base on model [7] and simulation [8] through the embedment of sensors in the vehicles. Sampling by most of these approaches were taken from actual driving test of vehicles in the real-world.

This study introduces an approach to estimate the instantaneous vehicle fuel consumption and $\mathrm{CO}_{2}$ emission from captured images by aerial traffic observation with a multi-rotor drone. With the benefits of mobility, accessibility, and flexibility from the drone to capture images from aerial observation over an actual traffic in almost any areas, this approach has the advantage of capturing the natural driving behaviour by many vehicles to understand the source of the pollutants from the varying traffic situations. The captured images are processed by tracking the movements of identified vehicles which are then used to predict the instantaneous vehicle dynamics, fuel consumption and $\mathrm{CO}_{2}$ emission through modelling. The modelling results were then compared with the vehicle fuel consumption measurement in understanding the challenges involved.

\section{METHODOLOGY}

The overall workflow of this study to estimate the vehicle fuel consumption and $\mathrm{CO}_{2}$ from aerial observation of drone is shown in Figure 1 which consist of four phases; aerial observation, image processing, vehicle modelling and analysis. 


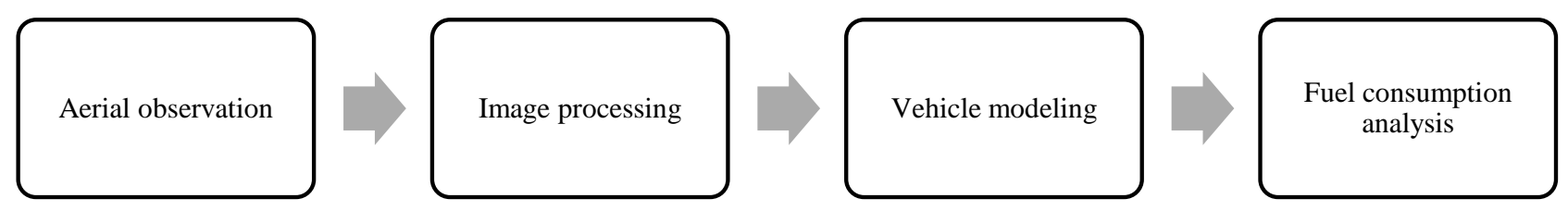

Figure 1 Overall worflow

\section{Aerial Observation}

The drone was deployed to capture high quality aerial video with the ability to provide stationary top views over the road traffic area. The camera on the drone captures the ground traffic footage at an altitude of $200 \mathrm{~m}$ above sea level with the camera oriented in a way that the optical axis is perpendicular to the ground surface to cover more area. This method also simplified the mathematic computation needed to compute the ground sample distance (GSD) [9]. As a case study, a four-way intersection with traffic lights as selected to be observed.

\section{Image Processing}

The drone was deployed to capture high quality aerial video with the ability to provide stationary top views over the road traffic area. The camera on the drone captures the ground traffic footage at an altitude of $200 \mathrm{~m}$ above sea level with the camera oriented in a way that the optical axis is perpendicular to the ground surface to cover more area. This method also simplified the mathematic computation needed to compute the ground sample distance (GSD) [9]. As a case study, a four-way intersection with traffic lights as selected to be observed.

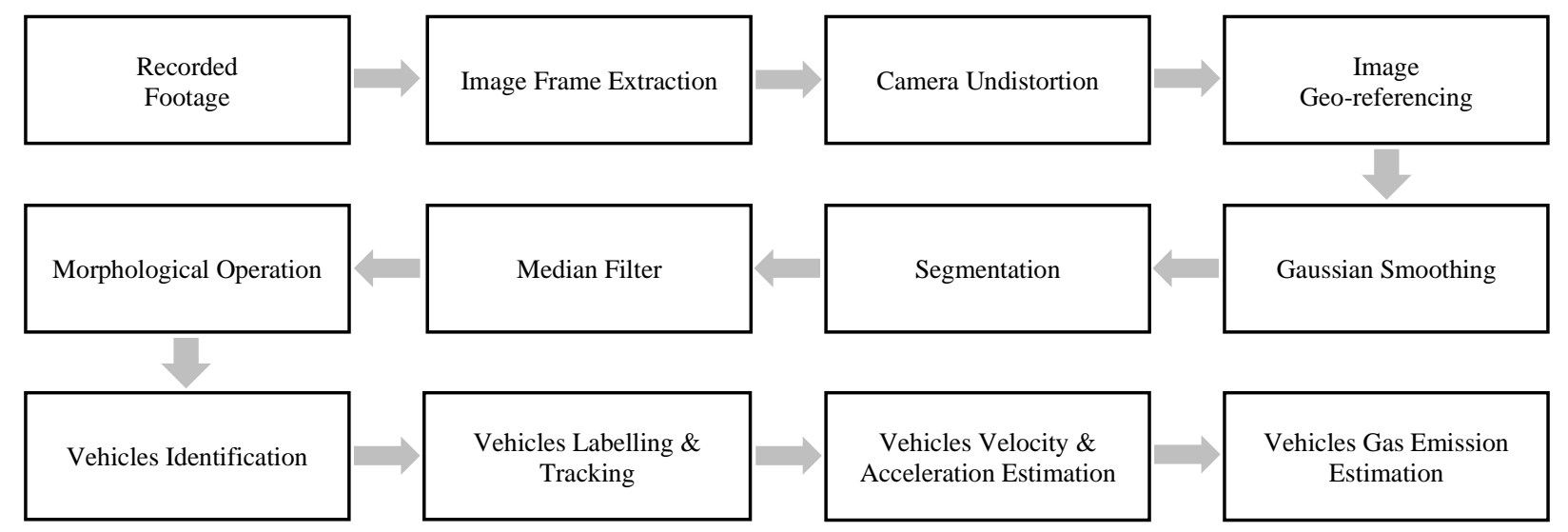

Figure 2 Workflow diagram of the image processing

It is crucial to understand the relationship between the captured image and the actual ground surface to estimate the global positioning system location of a vehicle in the recorded footage. The standard procedure for this matter is to orthorectify the image with the geo-referenced data [11]. From the orthorectified image, the ground-image relation is expressed using trigonometric principles, where Sw is camera sensor width, Iw is image width, h is the altitude of the drone above the ground and $\mathrm{f}$ is the focal length. The GSD, $\mathrm{P}$ is calculated using Equation 1 to estimate the actual distance on ground between two consecutive pixels.

$$
P=\frac{h \times S_{w}}{I_{w} \times f}
$$

The computation of vehicle speed is consisted of three steps. First step computes a set of features points of the vehicle for the first frames. Second step finds the matched feature points for two successive frame using optical flow technique. The final step computes the average speed by taking the average distance of the tracked feature points and divide it by the time difference between the frames. The distance between each tracked feature points is computed using Euclidian Equations 2 to 6 . Where $\Delta x(n), \Delta y(n)$ and $\Delta z(n)$ denotes difference of two feature point in $x-y-z$ axis.

$$
\begin{gathered}
\Delta_{x(n)}=\left|x_{2(n)}-x_{1(n)}\right| \times P \\
\Delta_{y(n)}=\left|y_{2(n)}-y_{1(n)}\right| \times P \\
\Delta_{z(n)}=\left|z_{2(n)}-z_{1(n)}\right|
\end{gathered}
$$




$$
\begin{gathered}
l_{n}=\sqrt{\Delta_{x(n)^{2}+\Delta_{y(n)}{ }^{2}++\Delta_{z(n)}{ }^{2}}} \\
v=\frac{\frac{1}{N} \sum_{1}^{N} l_{n}}{\Delta_{t}} \times 3.6 \quad \Delta_{t}=\frac{1}{F P S}
\end{gathered}
$$

\section{Vehicle Modelling}

This research uses a generic vehicle model using the common fundamentals of vehicle dynamics as shown Equation 7 where the traction force, $F_{t}$ is the sum of all resistance forces; inertia, aerodynamics, rolling and road grade. A quasistatic modelling approach was used to predict the instantaneous fuel consumption of a traffic based on the inputs from the image processing analysis. The quasistatic calculates the load power for the powertrain in backwards, from the wheels towards the power source. The wheels behaviour reacts to the actual vehicle activities which was obtained from the image processing. The total powertrain losses and fuel consumption were then determined by Equations 7 and 8, respectively.

$$
\begin{gathered}
F_{t}=\left(m_{v} \cdot \bar{a}_{i}\right)+\left(\frac{1}{2} \cdot \rho_{a} \cdot A_{f} \cdot c_{d} \cdot \bar{v}_{i}^{2}\right)+\left(c_{r} \cdot m_{v} \cdot g \cos \alpha_{i}\right)+\left(m_{v} \cdot g \sin \alpha_{i}\right) \\
\dot{m}_{f}=\bar{P}_{f} / H_{l}
\end{gathered}
$$

Where

$$
\bar{P}_{v}=\eta_{g} \cdot \eta_{a} \cdot \eta_{e} \cdot \bar{P}_{f}
$$

\section{Fuel Consumption Analysis}

The combustion model in this research applied the Willans approximation to estimate the fuel consumption for the required engine power. The Willans model as explained in [12] is a simplification by distinguishing the contributions of the thermodynamic and losses on the engine efficiency as shown in Equation 10. As for the transmission model, the average gear ratios based on the information from the selected top passenger cars were used.

$$
B M E P=M E P_{\text {fuel }} \cdot\left(\eta_{\text {comb }}\right)-F M E P
$$

Where

$$
\begin{gathered}
B M E P=\frac{T \cdot(4 \pi)}{V_{d}} \\
M E P_{\text {fuel }}=\frac{L H V \cdot m_{\text {burnt }}}{V_{d}} \\
\dot{m}_{\text {fuel }}=m_{\text {burnt }} \cdot \frac{N}{4 \pi}
\end{gathered}
$$

To initially observe the trend of the predicted instantaneous fuel consumption by the generic vehicle model with the actual measurement from author's previous study, a fixed driving condition of the NEDC test was used as the input into the model. The predicted instantaneous fuel consumption by the model shows similar trend with correlation coefficient of 0.872 . The total fuel economy predicted by the model is $7.418 \mathrm{~L} / 100 \mathrm{~km}$, higher by $3 \%$ from the experiment results.

\section{RESULTS AND DISCUSSION}

\section{Calculated Vehicle Speed and Acceleration}

The quality of the image by the drone camera was adequate for the pixel-based adaptive method to identify the vehicle pixels, allowing the top view area of the vehicle to be calculated in estimating the vehicle mass, frontal area, drag coefficient and engine displacement by the vehicle model. The program then labels and display the calculated instantaneous results of the tracked vehicle on the user-interface display. A test vehicle was used to understand the variations between the image processing results and the actual GPS measurement. Figure 3 shows the display of the image processing where the tracked vehicles are labelled with the estimated instantaneous speed, acceleration and fuel consumption. 


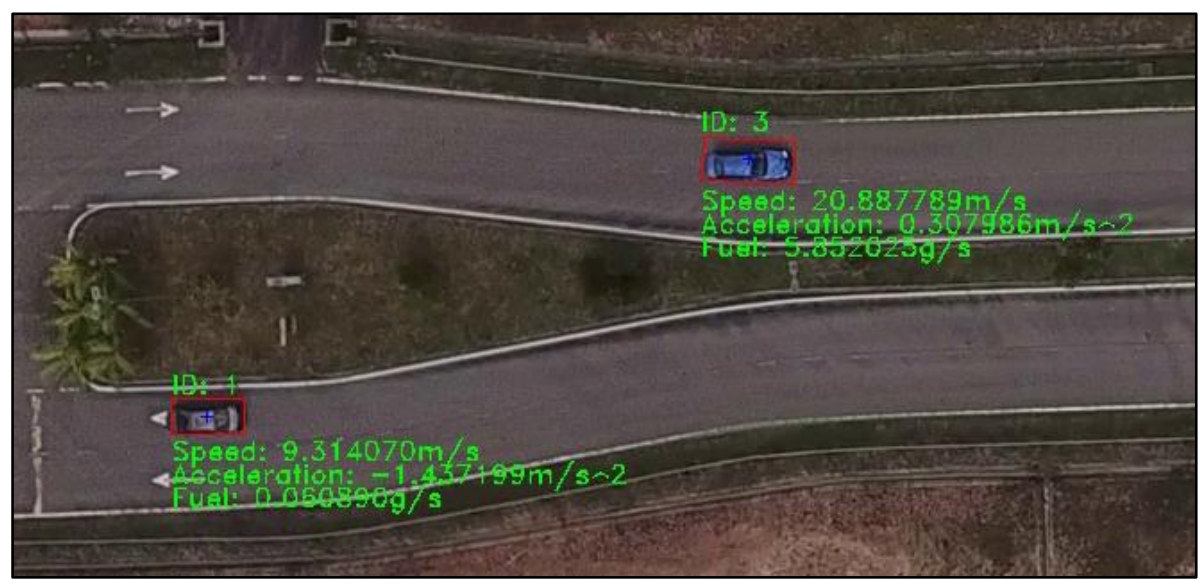

Figure 3 Vehicle tracking with instantaneous speed, acceleration and fuel consumption

The image processing calculates the vehicle travelling by estimating the ground sample distance which requires the drone to have good stabilization. However, the application of the commercial drone in this study has a limitation to provide enough stabilization precisely at the $200 \mathrm{~m}$ altitude against the wind. With movements and drifts by the drone affected by the wind, the calculated distance between two consecutive pixel centres measured on the ground by the image processing has affected the vehicle speed estimation. Figure 4 shows the variations of vehicle speed with the results from image processing higher by an average of $2.8 \mathrm{~m} / \mathrm{s}$ but may change according to the stability of the drone during the image recording. There is also a slight delay by the image processing to identify and track the vehicle. Due to the application of pixel-based adaptive approach, the program was only able to track the vehicle when the movement of the pixels shifts over a threshold, otherwise the pixels were treated as the background image. The vehicle needs to travel above $2.0 \mathrm{~m} / \mathrm{s}$ for the image processing to be able track the vehicle movements and estimate the vehicle speed. This causes a delay before the program starts to track, thus causes the sudden increase of calculated vehicle speed as it crosses the $2.0 \mathrm{~m} / \mathrm{s}$ threshold which explains the significant increase of calculated vehicle acceleration as shown in Figure 5.

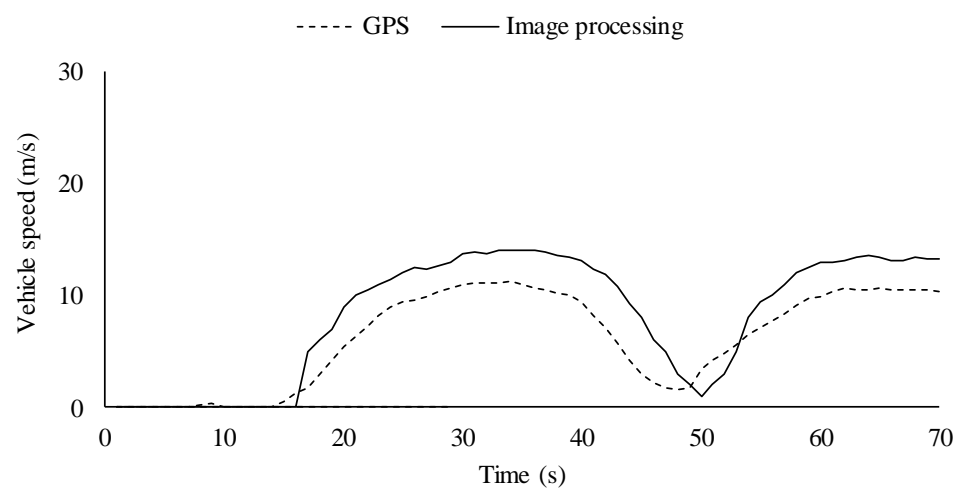

Figure 4 Vehicle speed profiles from GPS measurements and image processing

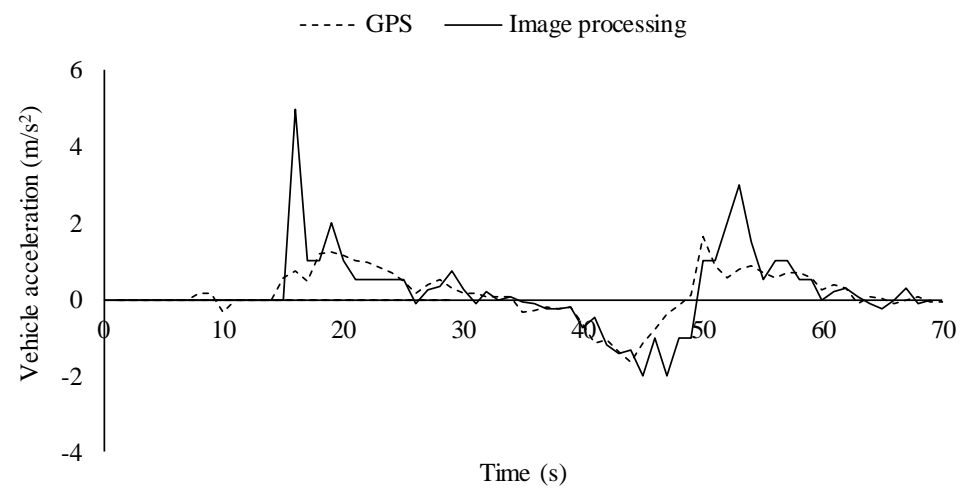

Figure 5 Vehicle acceleration profiles from GPS measurements and image processing 


\section{Calculated Fuel Consumption}

Figure 6 shows the comparison between the calculated fuel consumption with the actual measurement on the test vehicle. Figure 7 shows the estimated $\mathrm{CO}_{2}$ base on the instantaneous fuel consumption. On top of the variation by the vehicle model, the delay and offset from the image processing further contributed to the variations in the fuel consumption results. The higher calculated acceleration caused by the delay used as the input in the vehicle model contributed to the higher fuel consumption result. The estimated instantaneous fuel consumption by the program varies by an average of $0.168 \mathrm{~kg} / \mathrm{h}$ with correlation coefficient of 0.741 . Referring to the conversion by [13], the total $\mathrm{CO}_{2}$ emission was then calculated using the estimated fuel consumption. With such driving behaviour over the travelling distance of $0.573 \mathrm{~km}$, the total $\mathrm{CO}_{2}$ emission was calculated to be $2.68 \mathrm{~kg}$.

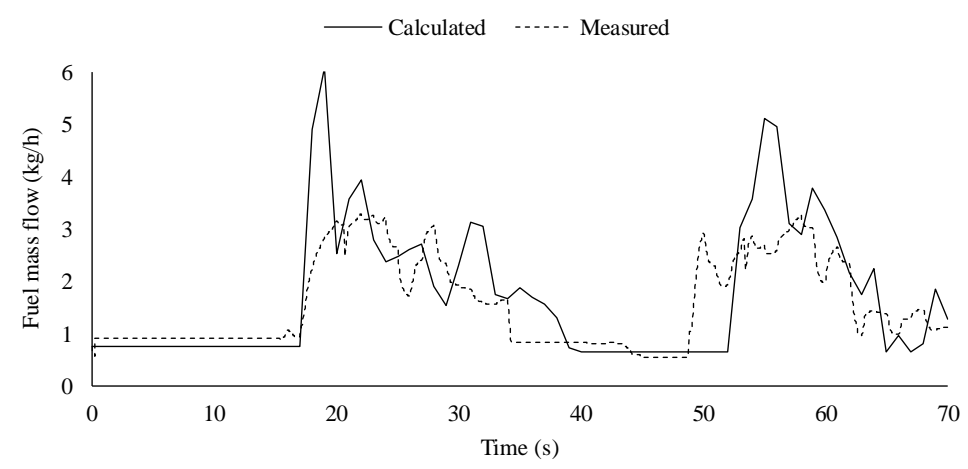

Figure 6 Fuel consumption comparison

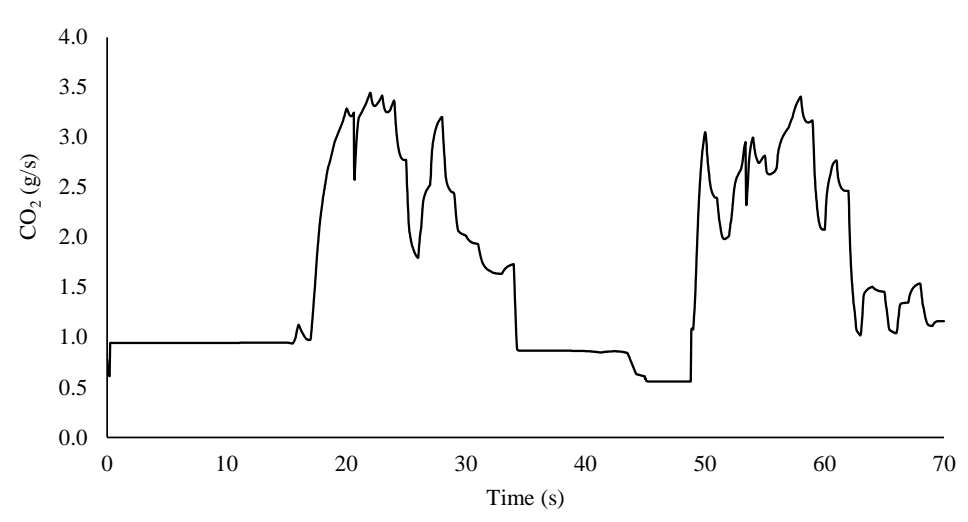

Figure 7 Calculated $\mathrm{CO}_{2}$ emission

\section{Case Study}

Figure 8 is the aeriel view capture by the drone while Figure 9 illustrates the distribution of the calculated total fuel used over the selected traffic area. The distribution of total fuel used was calculated by dividing the observed area into grids of $10 \mathrm{~m}^{2}$. The fuel consumption of vehicles that passed by each grid were accumulated to represent to the total fuel used within the period of the observation. The distribution shows highest fuel usage at location A and B. At location A, drivers were at an acceleration state before decelerating as they approached closer to intersection at location B either due to stop for the traffic light or entering the junction. Whereas at location B, drivers tend to accelerate while going through the intersection which may be due to the limited traffic light duration. Location $\mathrm{C}$ and $\mathrm{D}$ shows high fuel usage due to higher acceleration as they drove away from the intersection and at an acceleration state before they approached the intersection. 


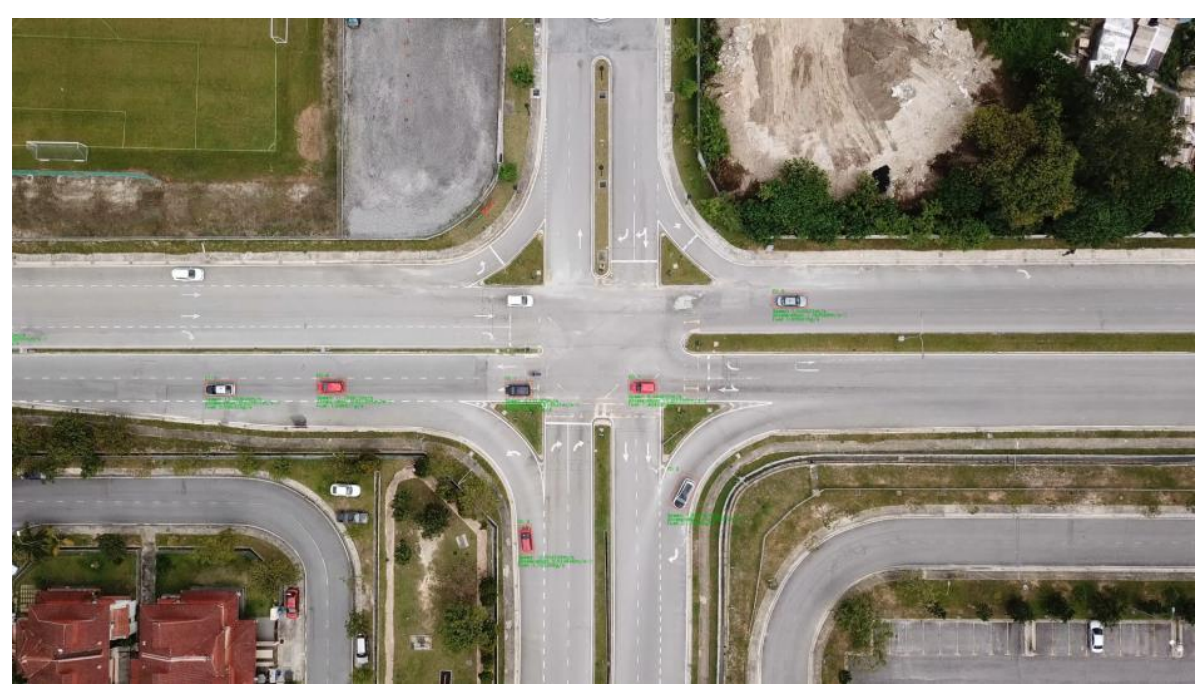

Figure 8 Aeriel view captured by the drone

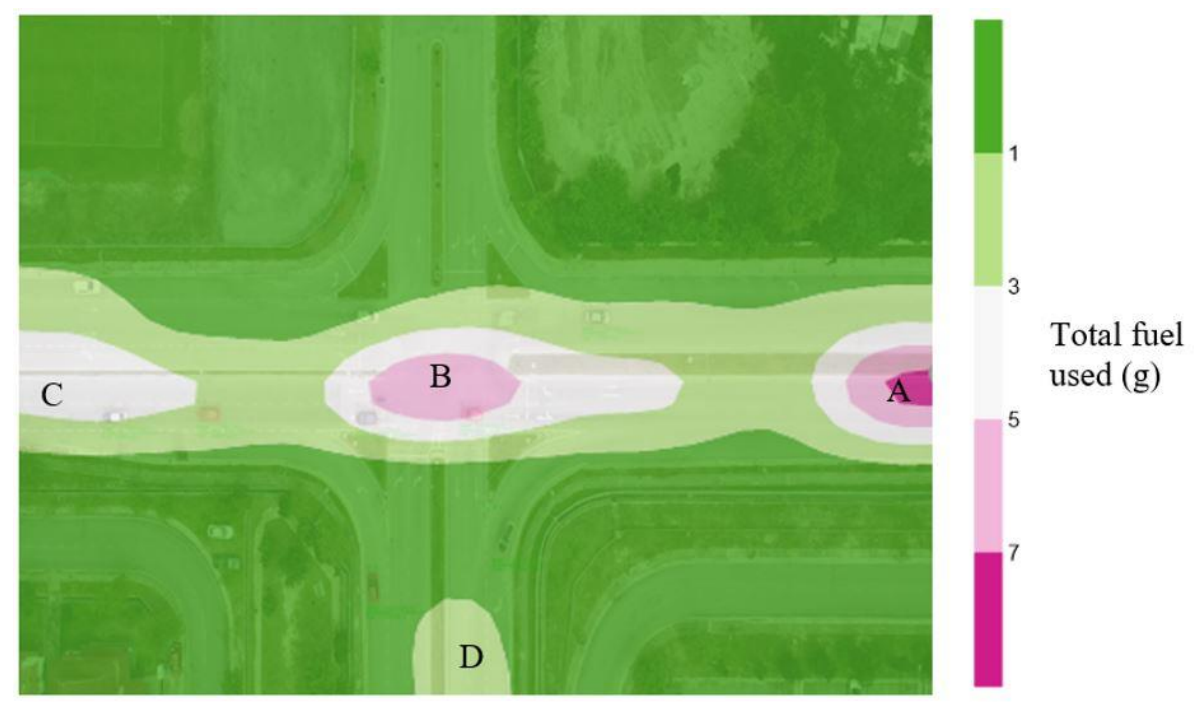

Figure 9 Distribution of total fuel used over the observed traffic

\section{CONCLUSION}

This study presents a preliminary approach of estimating the instantaneous fuel consumption from passenger car by aerial observation using a multi-rotor drone. The method of the proposed approach was presented, and variations of the estimated results were highlighted and discussed. The presented approach shows the capability of the image processing by aerial observation to estimate the traffic fuel consumption in relation to the natural driving behaviour without the needs to affect the vehicle and drivers. Further work will be required following to this stage of study through the improvements on the vehicle model, stability of the drone and application of machine learning in the image processing to achieve better estimation accuracy. The common application of machine learning approach instead of the pixel-based adaptive approach may further improves the performance of vehicle identification and tracking of the vehicle, allowing the recognition of vehicle type and model for further information to be used as the input for the vehicle model, thus improving the accuracy and reliability of the estimated results. The presented approach is believed to have the benefits over the mobility and scalability, potentially to complement the upcoming work in estimating instantaneous fuel consumption from actual road traffic.

\section{ACKNOWLEDGEMENT}

This work was supported/funded by the Ministry of Higher Education under Fundamental Research Grant Scheme (FRGS/1/2019/TK10/UTM/02/8).

\section{REFERENCES}

[1] K. Taylor, A. Post, T. B. Hoshizaki, and M. D. Gilchrist, "The effect of a novel impact management strategy on maximum 
[1] National Aeronautics and Space Administration. GISS Surface Temperature Analysis (GISTEMP v4). [cited 201902 Aug]; Available from: https://data.giss.nasa.gov/gistemp/.

[2] Khan, B. We Just Breached the 410 Parts Per Million Threshold. 2017 [cited 201902 Aug]; Available from: http://www.climatecentral.org/news/we-just-breached-the-410-parts-per-million-threshold-21372.

[3] ExxonMobil. Emission: All sectors contributing to restrain $\mathrm{CO}_{2}$ emissions growth. 2018 [cited 201902 Aug]; Available from: https://corporate.exxonmobil.com/en/Energy-and-environment/Energy-resources/Outlook-forEnergy/Emissions\#missionsrojections.

[4] Borken-Kleefeld, J. and T. Dallmann. (2018). Remote sensing of motor vehicle exhaust emissions. The International Council on Clean Transportation.

[5] Mahesh, S., Ramadurai, G., \& Nagendra, S. S. (2018). Real-world emissions of gaseous pollutants from diesel passenger cars using portable emission measurement systems. Sustainable Cities and Society, 41, 104-113.

[6] Triantafyllopoulos, G., Dimaratos, A., Ntziachristos, L., Bernard, Y., Dornoff, J., \& Samaras, Z. (2019). A study on the CO 2 and NOx emissions performance of Euro 6 diesel vehicles under various chassis dynamometer and on-road conditions including latest regulatory provisions. Science of The Total Environment, 666, 337-346.

[7] Kan, Z., Tang, L., Kwan, M. P., \& Zhang, X. (2018). Estimating vehicle fuel consumption and emissions using GPS big data. International journal of environmental research and public health, 15(4), 566.

[8] Jazcilevich, A. D., Garcia-Fragoso, A., Reynoso, A. G., Grutter, M., Diego-Ayala, U., Lents, J., \& Davis, N. (2007). A vehicle emissions system using a car simulator and a geographical information system: Part 1 - System description and testing. Journal of the Air \& Waste Management Association, 57(10), 1234-1240.

[9] Ericsson, E. (2000). Variability in urban driving patterns. Transportation Research Part D: Transport and Environment, 5(5): p. 337-354.

[10] Oro, D., Fernández, C., Saeta, J. R., Martorell, X., \& Hernando, J. (2011). Real-time GPU-based face detection in HD video sequences. In 2011 IEEE International Conference on Computer Vision Workshops (ICCV Workshops) (pp. 530-537). IEEE.

[11] Zhuo, X., Koch, T., Kurz, F., Fraundorfer, F., \& Reinartz, P. (2017). Automatic UAV image geo-registration by matching UAV images to georeferenced image data. Remote Sensing, 9(4), 376.

[12] Guzzella, L. and C. Onder. (2009) Introduction to modeling and control of internal combustion engine systems. Springer Science \& Business Media.

[13] Pinto, G. and M.T. Oliver-Hoyo. (2008). Using the relationship between vehicle fuel consumption and $\mathrm{CO}_{2}$ emissions to illustrate chemical principles. Journal of Chemical Education, 85(2): p. 218. 